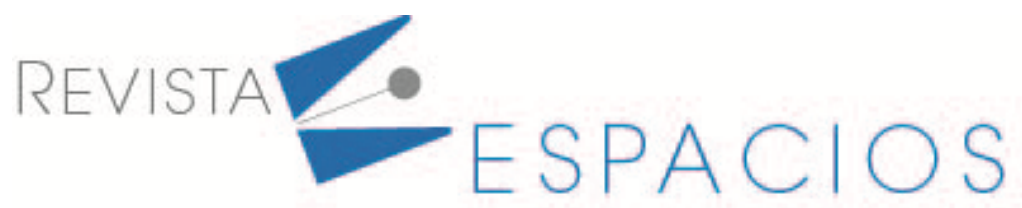

Vol. 42 (10) $2021 \cdot$ Art. 4

Recibido/Received: 08/02/2021 • Aprobado/Approved: 18/05/2021 • Publicado/Published: 30/05/2021

\title{
Una mirada a la reutilización del agua de lluvia en el Cantón Caldas del Ejército Nacional (Colombia)
}

\section{A glance at the re-use of rainwater in the Colombian National Army in the Canton of Caldas.}

\author{
LARA-BENAVIDES, Julián David ${ }^{1}$ \\ MONTOYA VARÓN, Néstor Freddy² \\ BAUTISTA-ZABALA, Leimer ${ }^{3}$ \\ PALACIO-GOMEZ, Diana Carolina ${ }^{4}$ \\ RICO-MARTÍNEZ, Mónica Andrea ${ }^{5}$
}

\begin{abstract}
Resumen
Se realizó un estudio de factibilidad del uso de agua de lluvia en las instalaciones del Cantón Militar Caldas, la creación de diseños preliminares para la reutilización de la misma y opciones de algunos dispositivos y métodos de tratamiento hídrico para disminuir el consumo de agua potable. Para esto se analizaron variables meteorológicas entre los años 2007 al 2017 y se practicaron muestreos y análisis de laboratorios del agua de lluvia e inspecciones a la red hidrosanitaria actual del cantón.

Palabras clave: escasez hídrica, reutilización de aguas de lluvias, sistema de uso eficiente del agua
\end{abstract}

\begin{abstract}
A feasibility study was carried out on the use of rainwater in the Caldas canton facilities, the creation of preliminary designs for its reuse and options for some devices and methods of water treatment to reduce the consumption of drinking water. For this, meteorological variables were analyzed between 2007 and 2017 and samples and laboratory analysis of rainwater were carried out and inspections of the current hydrosanitary network of the canton.

key words: water scarcity, reuse of rainwater, efficient water use system.
\end{abstract}

\section{Introducción}

El aprovechamiento del agua de lluvia es una práctica que ha tomado fuerza en los últimos años, ya que es ambientalmente sostenible y compensa el factor económico. En el contexto local de Bogotá se desconocen los beneficios de usar las aguas de lluvias, debido a que estas son desechadas y van al alcantarillado pluvial, que no tiene cubrimiento de toda la ciudad, o al combinado donde se une con el agua sanitaria y queda totalmente contaminada (Palacio, 2010). Al recolectar y usar el agua de lluvia se evita que esta finalice en el alcantarillado

\footnotetext{
1 Escuela de Ingenieros Militares. Colombia. julian.lara@esing.edu.co

2 Escuela de Ingenieros Militares. Colombia. fredd_891015@hotmail.com

3 Escuela de Ingenieros Militares. Colombia. Ibautistazabala@gmail.com

4 Escuela de Ingenieros Militares. Colombia. diana.palacio@esing.edu.co

5 Escuela de Ingenieros Militares. Colombia. moaricoma@unal.edu.co
} 
sin cumplir ningún uso, al mismo tiempo que se disminuye el consumo de agua potable. Esto genera que se reduzca la oferta del agua y por ende el estrés en las fuentes hídricas.

Es importante que en los cantones militares se empleen este tipo de alternativas para el ahorro en el consumo de agua potable, debido a que captar el agua pluvial para su reusó puede optimizar el ecosistema de una instalación mejorando la calidad de la relación de la edificación con el entorno. La reutilización de agua es una estrategia que permite la minimización de las aguas residuales, refinamiento de materias primas y procesos de producción y reutilización de los residuos (Jaramillo, 2010).

En la presente investigación se establece la Ingeniería conceptual en la cual se propone la modificación a la red hidrosanitaria existente con el fin de captar el agua de lluvia proveniente de las cubiertas de los edificios: Escuela de Ingenieros Militares (ESING), Comando de Ingenieros (COING) y el Edificio Lara, ubicados en el Cantón Militar Caldas en la ciudad de Bogotá D.C. Para esto se desarrollaron cuatro actividades principales:

1. Evaluar las características de la zona para determinar cómo son las codiciones hidrológicas particulares en el lugar; mediante la inspección a campo de las redes hidrosanitarias existentes en el Cantón Caldas y análisis de planos hidráulicos.

2. Detrminar cuál debe ser la calidad y cantidad apropiada del agua de lluvia, así como su variación en el año, para poder realizar un reemplazo total o parcial del agua potable, esto mediante análisis fisicoquímicos y estadísticos.

3. Establecer cuáles son los mejores dispositivos o métodos de tratamieto de agua a nivel predial a través de búsqueda bibliográfica y análisis de costos.

4. Generar un diseño preliminar del sistema de reutilización de agua de lluvia mediante cartografía y planos hidráulicos.

Este trabajo evidencia el resultado del proyecto de investigación científica aprobado por el Centro de Investigación de la ESING denominado: "Estudio de factibilidad para la reutilización de agua de lluvia en el cantón Caldas del Ejército Nacional. (FASE I)", el cual tiene como propósito principal diseñar un sistema de aprovechamiento de agua de lluvia en las instalaciones mencionadas. Por último con la implementación de este sistema de reutilización de aguas de lluvias se estaría haciendo un aporte importante a la gestión integral del recurso hídrico, como también a los costos asociados al consumo de agua potable en las instalaciones mencionadas suministrada por la empresa de servicios públicos de Bogotá.

\section{Metodología}

La metodología empleada en la presente investigación consta de 3 fases que se describen a continuación. 
Figura 1

Desarrollo metodológico

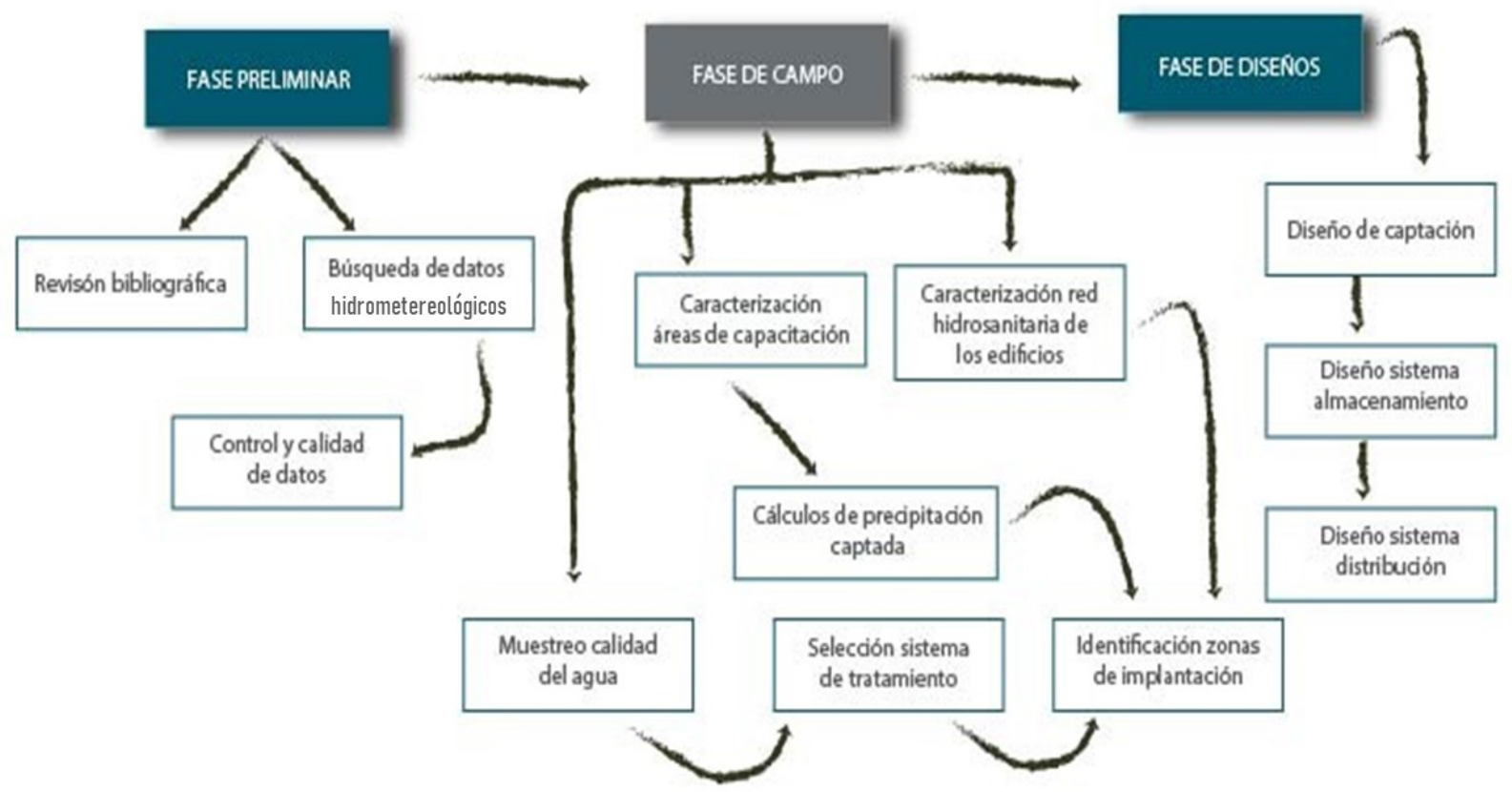

Fuente: Elaboración propia

\subsection{Fase Preliminar}

En esta etapa se realizó una revisión bibliográfica sobre el área de estudio en temas ligados a sus variables meteorológicas. También se revisaron conceptos sobre determinación de consumo de agua, sistemas de captación y almacenamiento de aguas de lluvias, posibles usos del agua de lluvia enfocado al cantón Caldas, búsqueda tecnológica a nivel local e internacional.

\subsection{Fase de Campo}

En esta etapa se realizó la revisión y análisis de datos meteorológicos entorno al área de estudio y la caracterización de esta. Así mismo, se caracterizaron las áreas de captación de agua en los edificios del Comando de Ingenieros (COING), la Escuela de Ingenieros Militares (ESING) y el Edificio Lara, así como la caracterización e inspección de la red hidrosanitaria de aguas de lluvias de dichos edificios. Se consultó con la división de Servicios Públicos del Ejercito Nacional para obtener el consumo histórico de agua potable del año 2017 y se realizaron la toma de análisis de laboratorio de las aguas de lluvia, mantiente la caracterización fisicoquímica que fue realizada por el laboratorio ANALQUIM LTDA, el cual está certificado por el Instituto de Hidrología, Meteorología y Estudios Ambientales (IDEAM), se hicieron ensayos para conocer \% de unidades en: Arsénico, cadmio, cobre, coliformes Totales, color, cromo, D.B.O. 5, D.Q.O, E. Coli, PH, sólidos Sedimentables, temperatura, mercurio, plomo, sólidos suspendidos totales, sólidos totales. Finalmente se realizó la identificación, diseño de tanques y selección utilizados en otros países, los cuales pueden ser una opción para implementarse en el proyecto.

\subsection{Fase de Análisis}

En esta etapa se realizó el análisis de homogenización de series de precipitación, se realizó una estimación de datos faltantes de precipitación diaria, para lo cual se tuvieron en cuenta los siguientes criterios: Cuenca hidrográfica, cinturón de condensación, índice de aridez, coeficiente de correlación y coeficiente de variación y 
se conjugó con los métodos de dobles masas y pruebas de bondad de ajuste (Kruskal Wallis). Se realizó la interpretación de los análisis de laboratorio de las aguas de lluvias y la interpretación y análisis de parámetros Turbiedad, pH, TDS, resistividad, conductividad y temperatura in situ.

\subsection{Análisis de datos preliminar}

\subsubsection{Selección e interpretación de Datos}

Para seleccionar e interpretar los datos se realizó una homogenización de series de precipitación, estas se identificaron mediante datos de la red de monitoreo de calidad del aire de Bogotá de la Secretaria Distrital de Ambiente de Colombia (SDA) (Secretaria Distrital de Ambiente, 2007). Para la homogenización estadística de datos se requiere, como condición básica, que éstos sean de la misma naturaleza, del mismo origen, obtenidos mediante observaciones y mediciones que hayan seguido los mismos procedimientos, métodos semejantes y la longitud de las series sean las mismas. En el estudio se utilizaron los datos diarios de precipitación desde el 2007 al 2017 y también se realizó una estimación de datos faltantes de precipitación diaria y se tuvieron en cuenta los siguientes criterios:

1. Cuenca hidrográfica: las estaciones que se tengan en cuenta para el relleno de datos deben estar en la misma cuenca, en este caso en la cuenca del río Bogotá.

2. Cinturón de codensación: las estaciones tenidas en cuenta para el relleno de datos se encuentran dentro del mismo cinturón de condensación (zona D) y esta zona pertenece también a la zona central, pero con influencia del transcurso caacterístico de la gran zona sur, por consiguiente el transcurso varia un poco y demuestra una traslación de un medio año del periodo principal anticiclónico que se presenta especialmente en las zonas altas, cuando la nubosidad de estancamiento sobrepasa las cimas y alcanza a extenderse hasta las partes altas de barlovento, como se conoce temporalmente por el tiempo reinante sobre la Sabana de Bogotá.

3. Índices de aridez (Lang) que es un dato fundamental las precipitaciones caídas a lo largo del año (como fuente de agua) y las temperaturas (como indicador de la capacidad para evaporar el clima).

4. Coerficiente de correlación: en el que se hace un análisis de dos variables y es posible determinar el grado de relación que tiene una respecto a la otra. Si se le llama a una variable $X$ y a la otra $Y$.

5. Coeficiente de variación: en las tablas 1 y 2 se calcularon las variables estadísticas tenidas en cuenta, para el relleno de las series de precipitación diaria.

El relleno de datos faltantes se inició con la estación que tenía la menor cantidad de vacíos, para el caso fue la estación de Puente Aranda la cual se rellenó utilizando la ecuación 3 y se complementó con la información e las estaciones : Carvajal Sevillana, Kennedy y Tunal, las cuales hacen parte del grupo 1, que cumplían con los criterios establecidos con anterioridad (ver tabla 1). Después de rellenar Puente Aranda se completan los datos de la siguiente estación que tenía menos datos faltantes y así sucesivamente.

Tabla 1

Variables estadísticas grupo 1

\begin{tabular}{|l|r|r|r|r|}
\hline Variable & $\begin{array}{l}\text { Puente } \\
\text { Aranda }\end{array}$ & $\begin{array}{l}\text { Carvajal } \\
\text { Sevillana }\end{array}$ & Kennedy & Tunal \\
\hline Ppromedio & 2,3 & 2,0 & 2,0 & 2,1 \\
\hline Cv & 2,3 & 2,3 & 2,4 & 2,2 \\
\hline Cs & 4,7 & 3,9 & 4,3 & 3,7 \\
\hline la & 0,2 & 0,1 & 0,1 & 0,1 \\
\hline Datos faltantes & \multicolumn{7}{|c|}{164} & 365 & 719 & 893 \\
\hline \multicolumn{7}{r|}{ Fuente: ESING }
\end{tabular}


El grupo 2 lo conforman las variables que cumplen con los criterios mencionados (ver tabla 2): Usaquén, Suba, Las Ferias y Puente Aranda, realizando en procedimiento ya mencionado.

Tabla 2

Variables estadísticas grupo 2

\begin{tabular}{|l|r|r|r|r}
\hline Variable & \multicolumn{1}{|l|}{ Las Ferias } & Suba & Usaquén & \multicolumn{2}{|l}{ Aranda } \\
\hline Ppromedio & 2,7 & 2,3 & 2,7 & 2,3 \\
\hline $\mathrm{Cv}$ & 2,2 & 2,2 & 2,3 & 2,3 \\
\hline $\mathrm{Cs}$ & 3,6 & 3,6 & 3,7 & 4,7 \\
\hline la & 0,2 & 0,2 & 0,2 & 0,2 \\
\hline Datos faltantes & 299 & 326 & 453 & 164 \\
\hline
\end{tabular}

Fuente: ESING

\subsubsection{Datos Meteorológicos}

Para el análisis de los datos meteorológicos, se tuvieron únicamente en cuenta las variables meteorológicas de precipitación y la temperatura media. Los datos fueron tomados de la estación automática Puente Aranda en un periodo de tiempo comprendido entre 2007 al 2017 (Secretaria Distrital de Ambiente, 2007), este periodo de 10 años permite recolectar y contrastar mejor los datos, tendiendo en cuenta que muchos de los puntos representan en algunos meses fallas y auncensias de datos, por lo cual se identifican varios como punto de partida en contrastar los datos.

En la figura 2 se registran dos fases húmedas, la primera de marzo a mayo y la segunda de octubre a diciembre, presentando la mayor precipitación en el mes de noviembre con $1473 \mathrm{~mm}$; la segunda fase es la de mayor precipitación, durante esos meses se realizarán los muestreos y análisis de laboratorio.

Figura 2

Histograma de la estación automática Puente Aranda

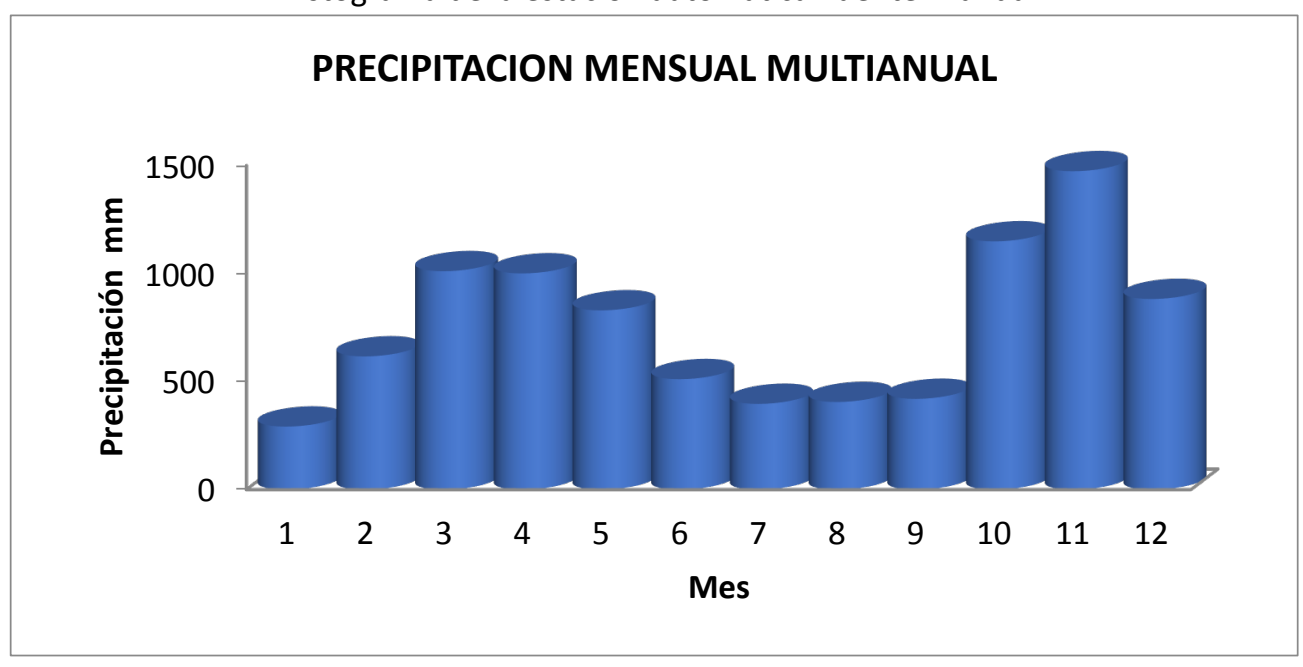

Fuente: ESING

\subsubsection{Red Hidrosanitaria Edificios ESING, COING y LARA}

La red hidrosanitaria de los edificios ESING, COING y LARA está conformada por sistemas independientes entre sí según lo plasmado en los planos hidráulicos e hidrosanitarios de cada edificio. Al realizar la inspección de la red hidrosanitaria de cada edificio, se determinó que los planos presentan diferencias e inconsistencias respecto a lo observado durante la inspección. Por esta razón se realizará la medición y toma de puntos hidrosanitarios con el objetivo de establecer la red de recolección de las aguas de lluvias. En el recorrido se evidenció que para 
el edificio ESING y COING esta red y los bajantes de agua de lluvia se encuentran en el sótano, y sus puntos de salida conducen a las cajas de inspección, como se observa en las figuras 3 ( $a$, b y c):

Figura 3

Puntos salida red aguas de lluvias edificio COING. A) Costado Suroriental B) sector oeste. C Entrega red aguas de lluvias y pozos eyectores edificio COING. D) Entrega aguas de lluvias edificio LARA costado sur-oriental.

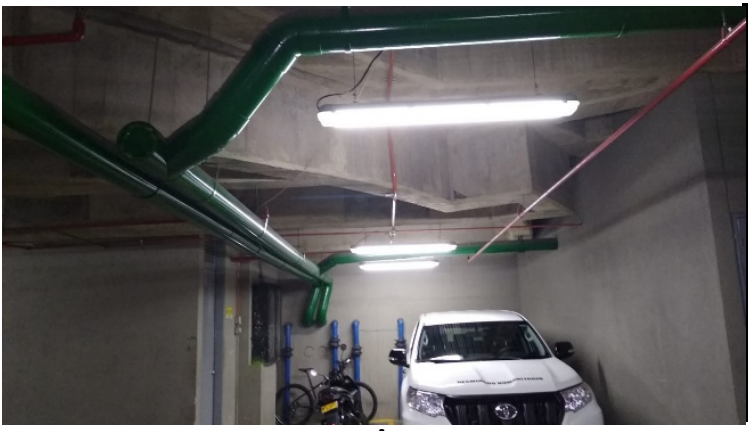

A

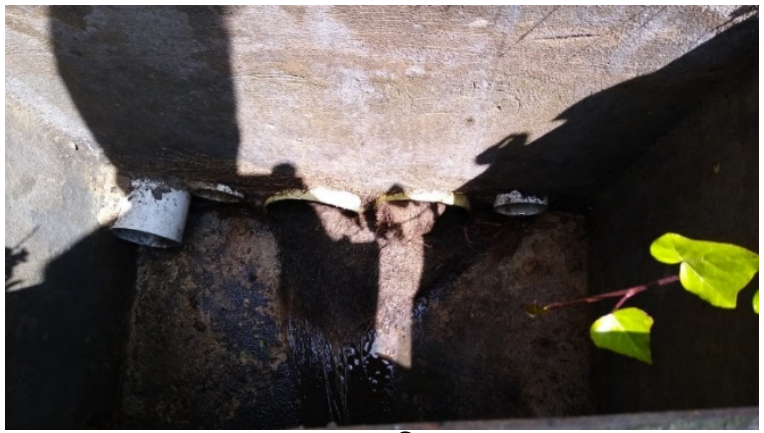

C

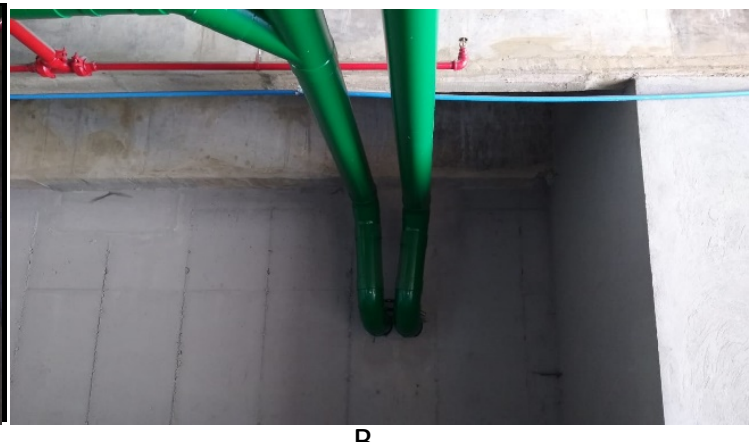

B

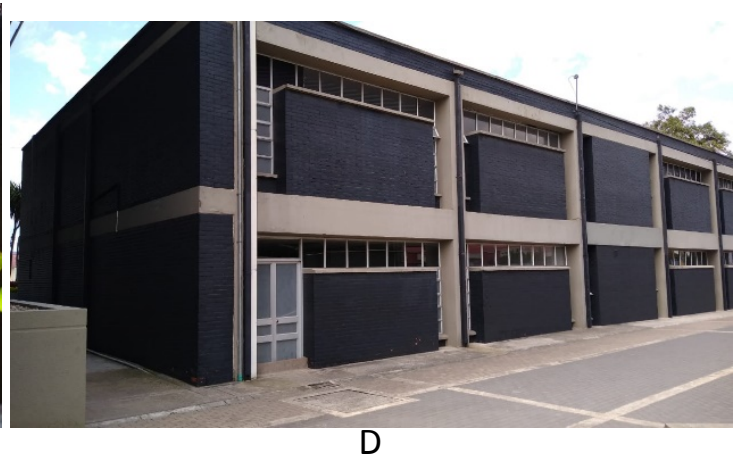

D

Fuente: ESING

Para el edificio LARA se realizaron las mediciones en la parte exterior, ya que el desagüe de la localiza a nivel de la cubierta. Adicionalmente se evidenció que la entrega de las bajantes es de tipo superficial, ya que estas entregan sobre el andén por el costado Noroccidental, y a una cuneta por el costado Sur-oriental como se observa en las figuras 3 (d).

\section{Resultados y discusión}

\subsection{Calidad de agua y consumo}

En cuanto al consumo de agua, se consultó con la división de Servicios Públicos del Ejército Nacional para obtener el consumo histórico de agua potable del año 2017; sin embargo se encontró que los edificios no cuentan con un contador independiente. Al encontrarse al interior del Cantón Caldas, la facturación que llega por parte de la Empresa de Acueducto de Bogotá EAAB no distingue el consumo individual de casa edificio.

Teniendo en cuenta el área de estudio que presenta una altura promedio de 2600 m.s.n.m, se infiere una dotación neta de 120 L/(hab-día) de acuerdo la ecuación 1. El porcentaje de pérdidas máximas aceptadas por la norma son del $25 \%$, este valor de pérdidas está asociado a una mayor ración de la dotación bruta, sin embargo el uso de los edificios es de oficina y no de vivienda, por lo que el uso de agua se encuentra destinada en su mayoría para baños y limpieza, de tal manera que se van a asumir pérdidas del $0 \%$. 


$$
\text { Dotación Bruta }=\frac{120 \frac{L}{h a b-d i ́ a}}{1}=\frac{120 L}{\text { hab-día }}
$$

A partir de los planos arquitectónicos de los edificios en los cuales se encuentra la información de la cantidad de trabajadores y estudiantes para la cual fue diseñado cada edificio, se establece la cantidad de personas que deben ser abastecidas por edificio como se puede observar en la tabla 4.

Tabla 4

Estimación de la población de los edificios de estudio.

\begin{tabular}{|c|c|}
\hline Edificio & Población aproximada \\
\hline Comando de Ingenieros (COING) & 745 \\
\hline Escuela de Ingenieros (ESING) & 996 \\
\hline Edificio Lara & 78 \\
\hline
\end{tabular}

Fuente: ESING

A partir de la información obtenida de los planos, y la dotación bruta por persona, se asigna el consumo requerido para cada uno de los edificios este análisis se obtiene mediante las ecuaciones 2 , 3, y 4 :

Escuela de Ingenieros (ESING):

$$
\text { Consumo }=996 \text { hab } * 120 \frac{L}{h a b-\text { día }} * \frac{30 \text { días } * m^{3}}{1000 \mathrm{~L} * \text { mes }}=3585.6 \frac{\mathrm{m}^{3}}{\mathrm{mes}}(2)
$$

Comando de Ingenieros (COING):

Edificio Lara:

$$
\text { Consumo }=745 h a b * 120 \frac{L}{\text { hab-día }} * \frac{30 \text { días } * m^{3}}{1000 \text { L*mes }}=2682 \frac{\mathrm{m}^{3}}{\text { mes }}(3)
$$

$$
\text { Consumo }=78 h a b * 120 \frac{L}{h a b-d i ́ a} * \frac{30 \text { días } * m^{3}}{1000 \text { L*mes }}=280.8 \frac{m^{3}}{m e s}(4)
$$

Actualmente los edificios no se utilizan en su totalidad, se planteó que solo hay un $40 \%$ de la población máxima de cada uno de los edificios. Según la RAS 0330/17 se recomienda que la dotación máxima para Bogotá sea de 120 L/hab*día, esto se hace a partir de estudios históricos realizados por las empresas prestadoras de los servicios de acueducto, la siguiente tabla 5 da el valor máximo a partir de la altura del sitio que se quiera estudiar, Bogotá se encuentra a 2630 m.s.n.m, por lo cual se toma la primera opción.

Tabla 5

Uso y capacidad actual de los edificios

\begin{tabular}{|c|c|c|}
\hline Edificio & Total Habitantes & Uso (Habitantes) \\
\hline ESING & 996 & $40 \%=400$ \\
\hline COING & 745 & $40 \%=300$ \\
\hline LARA & 78 & 78 \\
\hline & TOTAL & 778 \\
\hline
\end{tabular}

Fuente: ESING

Por medio de los planos proporcionados por topografía se halló que el área total del cantón es de $183094 \mathrm{~m}^{2}$, pero su área útil es de $159355.84 \mathrm{~m}^{2}$, se restaron las zonas donde se pierde el agua de lluvia por filtración en adoquín o porque hay zonas verdes que no permiten la captación del agua. Por complicaciones en los diseños de instalaciones sanitarias y de alcantarillado en el Cantón no se puede recolectar el 100\% de la precipitación, por lo cual solo se tomó el área útil alrededor de los tres edificios y es de aproximadamente $8000 \mathrm{~m}^{2}$. Actualmente se cuenta con un tanque de recolección de agua de lluvia de $117 \mathrm{~m}^{3}$ en el edificio COING, que se usa para abastecer las instalaciones de incendios, el tanque de agua potable tienen una capacidad de $37 \mathrm{~m}^{3}$. Se tomaron 
en promedio 10 horas laborales diarias, para un consumo de 50 litros de agua diarios y un promedio de 25 días laborales por mes y se desarrolló mediante la ecuación 5.

$$
D=\frac{778 * 25 * 50}{1000}=972.5 \mathrm{~m}^{3} / \text { mes (5) }
$$

El uso principal de los edificios es el de oficinas, en las cuales sus habitantes no pasan las 24 horas del día; por tal motivo el personal no consumirán los $120 \mathrm{~L}$ de agua per cápita.

$$
\text { Consumo por hora }=\frac{120 \mathrm{~L}}{24 \text { horas }}=5 \frac{\mathrm{L}}{\text { hora }}(6)
$$

Sin embargo es necesario identificar cuanto seria el consumo de agua teniendo cuen cuenta el numero de personas que hacen uso del recurso, para esto se tomarán en promedio 10 horas laborales diarias, un consumo de 50 litros de agua diarios y un promedio de 25 días laborales por mes, como lo demuestran las ecuaciones 7, 8 y 9 :

Comando de Ingenieros (COING):

$$
\text { Consumo }=996 \text { hab } * 50 \frac{L}{\text { hab-día laboral }} * \frac{25 \text { días laborales } * m^{3}}{1000 \text { L } * \text { mes }}=1245 \frac{\mathrm{m}^{3}}{\mathrm{mes}}(7)
$$

Escuela de Ingenieros (ESING):

$$
\text { Consumo }=745 \text { hab } * 50 \frac{L}{h a b-\text { día }} * \frac{25 \text { días laborales } * m^{3}}{1000 \text { L*mes }}=931.25 \frac{\mathrm{m}^{3}}{\mathrm{mes}}(8)
$$

Edificio Lara:

$$
\text { Consumo }=78 h a b * 50 \frac{L}{\text { hab-día }} * \frac{25 \text { días laborales } * m^{3}}{1000 \text { L*mes }}=97.5 \frac{\mathrm{m}^{3}}{\text { mes }}(9)
$$

Por otro lado para saber cuál es la oferta de agua de lluvia se realiza mediante la ecuación 10, entendiéndose esta como: $A=$ oferta mensual $\left(\mathrm{m}^{3}\right) P p=$ precipitación promedio mensual $\left(\mathrm{L} / \mathrm{m}^{2}\right) \mathrm{Ce}=$ coeficiente de escorrentía. (Teja metálica $=0,9) A c=$ área de captación.

$$
\begin{gathered}
A=\frac{P p * C e * A c}{1000}(10) \\
A=\frac{857.9 * 0,9 * 8000}{1000}=6177 \mathrm{~m}^{3}
\end{gathered}
$$

Para conocer el volumen de agua de lluvia a recolectar se necesita tomar a la precipitación del mes de noviembre por ser este el mes donde se presentaron mayores volúmenes de agua, evaluando el período comprendido entre el 2007 a 2017 y se evidencia mediante la aplicación de la ecuación 11.

$$
V=A-D \quad V=6177-2801=3376 \mathrm{~m}^{3}(11)
$$

En el siguiente cálculo se toma como coeficiente de escorrentía $\mathrm{C}=1$, ya que las superficies de la cubierta al ser teja y concreto, se consideran como material impermeable. Es por eso que se aplicó la ecuación $Q=C I A$ Dónde: $\mathrm{Q}=$ Caudal I= Intensidad A= área y desarrollas en las ecuaciones 12, 13 y 14 reflejadas en la Tabla 6. 
Tabla 6

Caudal requerido por edificio

\begin{tabular}{|c|c|}
\hline EDIFICIO & CAUDAL REQUERIDO \\
\hline Escuela de Ingenieros (ESING) & $Q=\frac{1 *\left(112.5 \frac{\mathrm{mm}}{\mathrm{mes}}\right) * \mathbf{9 1 6 . 9 4 6 \mathrm { m } ^ { 2 }}}{\mathbf{1 0 0 0}}=\mathbf{1 0 3 . 1 6} \frac{\mathrm{m}^{3}}{\mathrm{mes}}(12)$ \\
\hline Comando de ingenieros (COING) & $Q=\frac{1 *\left(112.5 \frac{\mathrm{mm}}{\mathrm{mes}}\right) * 1854.96 \mathrm{~m}^{2}}{1000}=\mathbf{2 0 8 . 6 8} \frac{\mathrm{m}^{3}}{\mathrm{mes}}(13)$ \\
\hline Edificio Lara & $Q=\frac{1 *\left(112.5 \frac{\mathrm{mm}}{\mathrm{mes}}\right) * \mathbf{6 2 7 . 1 9 3 m ^ { 2 }}}{\mathbf{1 0 0 0}}=\mathbf{6 0 . 1} \frac{\mathrm{m}^{3}}{\mathrm{mes}}(14)$ \\
\hline
\end{tabular}

Fuente: ESING

\subsection{Análisis de laboratorio de las aguas de lluvias del área de estudio}

Para la reutilización del agua de lluvia es necesario realizar una caracterización fisicoquímica de la misma. La medición de los diferentes parámetros fue realizada por el laboratorio ANALQUIM LTDA, el cual está certificado por el Instituto de Hidrología, Meteorología y Estudios Ambientales (IDEAM). Con los resultados conseguidos, se realizó una comparación con los estandares nacionales definidos en la columna tres (limites de cuantificación). La sensibilidad de los equipos de ANALQUIM LTDA, presenta los límites de cuantificación de los parámetros de calidad de agua para las pruebas in situ y pruebas de laboratorio. Los resultados obtenidos (ver tablas 7, 8 y 9) se comparan con la Resolución 2115 del 2007 del Ministerio de la Protección Social y el Ministerio de Ambiente, Vivienda y Desarrollo Territorial (Ministerio de la proteccion social y de ambiente, 2007), la cual proporciona los valores aceptables de los parámetros fisicoquímicos y microbiológicos del agua apta para consumo humano, se comparan valores con parámetros de agua potable debido a que el sistema de tuberías de suministro de agua a los edificios es el mismo para los baños que para la cocina. La empresa ANALQUIM LTDA. utilizó el método de Sustrato enzimático-Multicelda para el análisis de coliformes totales, siguiendo la guía del IDEAM para la determinación de coliformes totales y E. coli por el método NMP (Número Más Probable), la guía del IDEAM se basa en el decreto 475 de 1998, con un valor aceptable para agua potable por número más probable de 0 microorganismos $/ 100 \mathrm{~mL}$.

Tabla 7

Resultados calidad de agua

de lluvia del edificio ESING

\begin{tabular}{|c|c|c|c|c|}
\hline Ensayo & Unidades & $\begin{array}{c}\text { Límites de } \\
\text { Cuantificación }\end{array}$ & Resultado & Criterio \\
\hline Arsénico & $\mathrm{mg} / \mathrm{L} \mathrm{Cd}$ & $<0.01$ & $<0.005 \mathrm{mg} / \mathrm{L}$ As & $\begin{array}{l}\text { Cumple con la resolución } 2115 \text {, el valor máximo } \\
\text { aceptado es de } 0.01 \mathrm{mg} / \mathrm{L} \text { As, y el resultado está } \\
\text { por debajo del límite de cuantificación del } \\
\text { instrumento con el que se realizó el ensayo. }\end{array}$ \\
\hline Cadmio & $\mathrm{mg} / \mathrm{L} \mathrm{Cu}$ & 0.05 & $<0.01 \mathrm{mg} / \mathrm{L} \mathrm{Cd}$ & $\begin{array}{l}\text { Cumple con la resolución } 2115 \text {, el valor máximo } \\
\text { aceptado es de } 0.003 \mathrm{mg} / \mathrm{L} \mathrm{Cd} \text {, y el resultado está } \\
\text { por debajo del límite de cuantificación del } \\
\text { instrumento con el que se realizó el ensayo. }\end{array}$ \\
\hline Cobre & $\mathrm{NMP} / 100 \mathrm{~mL}$ & 1.8 & $<0.05 \mathrm{mg} / \mathrm{L} \mathrm{Cu}$ & $\begin{array}{l}\text { Cumple con la resolución } 2115 \text {, el valor máximo } \\
\text { aceptado es de } 1.0 \mathrm{mg} / \mathrm{L} \mathrm{Cu} \text {, y el resultado está por } \\
\text { debajo del límite de cuantificación del instrumento } \\
\text { con el que se realizó el ensayo. }\end{array}$ \\
\hline $\begin{array}{c}\text { Coliformes } \\
\text { Totales }\end{array}$ & UPC & 5 & $\begin{array}{c}1.012 \times 10^{4} \\
\mathrm{NMP} / 100 \mathrm{~mL}\end{array}$ & $\begin{array}{l}\text { No cumple con el valor aceptable para agua } \\
\text { potable de acuerdo al decreto } 475 \text { de } 1998 \\
\text { (Ministerio de Salud Publica, 1998) y con la } \\
\text { resolución } 2115 \text { de } 2007 \text { (Ministerio de la } \\
\text { proteccion social y de ambiente, 2007) }\end{array}$ \\
\hline
\end{tabular}




\begin{tabular}{|c|c|c|c|c|}
\hline Ensayo & Unidades & $\begin{array}{c}\text { Límites de } \\
\text { Cuantificación }\end{array}$ & Resultado & Criterio \\
\hline Color & $\mathrm{mg} / \mathrm{L} \mathrm{Cr}$ & 0.05 & 12 UPC & $\begin{array}{l}\text { Esta característica física está por debajo del valor } \\
\text { máximo permisible en la resolución } 2115 \text {, por } \\
\text { tanto cumple el criterio de calidad de agua } \\
\text { potable. }\end{array}$ \\
\hline Cromo & $\mathrm{mg} / \mathrm{L} \mathrm{O} 2$ & 2 & $<0.05 \mathrm{mg} / \mathrm{L} \mathrm{Cr}$ & $\begin{array}{l}\text { Cumple con la resolución } 2115 \text {, el valor máximo } \\
\text { aceptado es de } 0.05 \mathrm{mg} / \mathrm{L} \mathrm{Cr} \text {, y el resultado está } \\
\text { por debajo del límite de cuantificación del } \\
\text { instrumento con el que se realizó el ensayo. }\end{array}$ \\
\hline $\mathrm{DBO}_{5}$ & $\mathrm{mg} / \mathrm{L} \mathrm{O} 2$ & 10 & $5 \mathrm{mg} / \mathrm{L} \mathrm{O}_{2}$ & \\
\hline $\mathrm{DQO}$ & $\mathrm{NMP} / 100 \mathrm{~mL}$ & 1 & $40 \mathrm{mg} / \mathrm{L} \mathrm{O}$ & \\
\hline E. Coli & Unidades & --- & $\begin{array}{l}6.1 \times 10^{1} \\
\mathrm{NMP} / 100 \mathrm{~mL}\end{array}$ & $\begin{array}{l}\text { No cumple con el valor aceptable para agua } \\
\text { potable de acuerdo al decreto } 475 \text { de } 1998 \text {, y con } \\
\text { la resolución } 2115 \text { de } 2007 \text {. }\end{array}$ \\
\hline $\mathrm{pH}$ & $\mathrm{mL} / \mathrm{L}$ & 0.1 & $\begin{array}{c}7.63 \text { Unidades de } \\
\mathrm{pH}\end{array}$ & \\
\hline $\begin{array}{l}\text { Sólidos } \\
\text { Sedimenta } \\
\text { bles }\end{array}$ & ${ }^{\circ} \mathrm{C}$ & --- & $0.2 \mathrm{~mL} / \mathrm{L}$ & \\
\hline $\begin{array}{c}\text { Temperatu } \\
\text { ra }\end{array}$ & $\mathrm{mg} / \mathrm{L} \mathrm{Hg}$ & 0.002 & $16.0^{\circ} \mathrm{C}$ & ----- \\
\hline Mercurio & $\mathrm{mg} / \mathrm{LPb}$ & 0.02 & $<0.002 \mathrm{mg} / \mathrm{L} \mathrm{Hg}$ & $\begin{array}{l}\text { Cumple con la resolución } 2115 \text {, el valor máximo } \\
\text { aceptado es de } 0.001 \mathrm{mg} / \mathrm{L} \mathrm{Hg} \text {, y el resultado está } \\
\text { por debajo del límite de cuantificación del } \\
\text { instrumento con el que se realizó el ensayo. }\end{array}$ \\
\hline Plomo & $\mathrm{mg} / \mathrm{L}$ & 5 & $<0.02 \mathrm{mg} / \mathrm{L} \mathrm{Pb}$ & $\begin{array}{l}\text { Cumple con la resolución } 2115 \text {, el valor máximo } \\
\text { aceptado es de } 0.01 \mathrm{mg} / \mathrm{L} \mathrm{Pb} \text {, y el resultado está } \\
\text { por debajo del límite de cuantificación del } \\
\text { instrumento con el que se realizó el ensayo. }\end{array}$ \\
\hline $\begin{array}{c}\text { Sólidos } \\
\text { suspendid } \\
\text { os totales }\end{array}$ & $\mathrm{mg} / \mathrm{L}$ & 35 & $<0.5 \mathrm{mg} / \mathrm{L}$ & \\
\hline $\begin{array}{l}\text { Sólidos } \\
\text { totales }\end{array}$ & 400 & 401 & $402 \mathrm{~g} / \mathrm{L}$ & \\
\hline
\end{tabular}

Fuente: Escuela de Ingenieros Militares (ESING)

\section{Resultados para ensayos realizados a la muestra de agua tomada en el Sótano COING.}

Tabla 8

Resultados calidad de agua

de lluvia del edificio COING

\begin{tabular}{|c|c|l|}
\hline Ensayo & Resultado & \multicolumn{1}{c|}{ Criterio } \\
\hline Arsénico & $<0.005 \mathrm{mg} / \mathrm{L} \mathrm{As}$ & $\begin{array}{l}\text { Cumple con la resolución 2115, el valor máximo aceptado es de } \\
0.01 \mathrm{mg} / \mathrm{L} \mathrm{As} \text {, y el resultado está por debajo del límite de cuantificación } \\
\text { del instrumento con el que se realizó el ensayo. }\end{array}$ \\
\hline Cadmio & $<0.01 \mathrm{mg} / \mathrm{L} \mathrm{Cd}$ & $\begin{array}{l}\text { Cumple con la resolución 2115, el valor máximo aceptado es de } \\
0.003 \mathrm{mg} / \mathrm{L} \mathrm{Cd}, \text { y el resultado está por debajo del límite de cuantificación } \\
\text { del instrumento con el que se realizó el ensayo. }\end{array}$ \\
\hline Cobre & $<0.05 \mathrm{mg} / \mathrm{L} \mathrm{Cu}$ & $\begin{array}{l}\text { Cumple con la resolución 2115, el valor máximo aceptado es de } \\
1.0 \mathrm{mg} / \mathrm{L} \mathrm{Cu} \text { y el resultado está por debajo del límite de cuantificación } \\
\text { del instrumento con el que se realizó el ensayo. }\end{array}$ \\
\hline Coliformes Totales & $4.02 \times 10^{3} \mathrm{MP} / 100 \mathrm{~mL}$ & $\begin{array}{l}\text { No cumple con el valor aceptable para agua potable de acuerdo al } \\
\text { decreto 475 de 1998, y con la resolución 2115 de 2007. }\end{array}$ \\
\hline
\end{tabular}




\begin{tabular}{|c|c|c|}
\hline Ensayo & Resultado & Criterio \\
\hline Color & $<5$ UPC & $\begin{array}{l}\text { Esta característica física está por debajo del valor máximo permisible } \\
\text { en la resolución } 2115 \text {, por tanto cumple el criterio de calidad de agua } \\
\text { potable. }\end{array}$ \\
\hline Cromo & $<0.05 \mathrm{mg} / \mathrm{L} \mathrm{Cr}$ & $\begin{array}{l}\text { Cumple con la resolución } 2115 \text {, el valor máximo aceptado es de } \\
0.05 \mathrm{mg} / \mathrm{L} \mathrm{Cr} \text {, y el resultado está por debajo del límite de cuantificación } \\
\text { del instrumento con el que se realizó el ensayo. }\end{array}$ \\
\hline $\mathrm{DBO}_{5}$ & $4 \mathrm{mg} / \mathrm{L} \mathrm{O}_{2}$ & \\
\hline DQO & $29 \mathrm{mg} / \mathrm{L} \mathrm{O}_{2}$ & \\
\hline E. Coli & $1.203 \times 10^{3} \mathrm{NMP} / 100 \mathrm{~mL}$ & $\begin{array}{l}\text { No cumple con el valor aceptable para agua potable de acuerdo al } \\
\text { decreto } 475 \text { de } 1998 \text {, y con la resolución } 2115 \text { de } 2007 \text {. }\end{array}$ \\
\hline $\mathrm{pH}$ & 6.58 Unidades de $\mathrm{pH}$ & \\
\hline $\begin{array}{c}\text { Sólidos } \\
\text { Sedimentables }\end{array}$ & $0.1 \mathrm{~mL} / \mathrm{L}$ & \\
\hline Temperatura & $18.0^{\circ} \mathrm{C}$ & \\
\hline Mercurio & $<0.002 \mathrm{mg} / \mathrm{L} \mathrm{Hg}$ & $\begin{array}{l}\text { Cumple con la resolución } 2115 \text {, el valor máximo aceptado es de } \\
0.001 \mathrm{mg} / \mathrm{L} \mathrm{Hg} \text {, y el resultado está por debajo del límite de cuantificación } \\
\text { del instrumento con el que se realizó el ensayo. }\end{array}$ \\
\hline Plomo & $<0.02 \mathrm{mg} / \mathrm{L} \mathrm{Pb}$ & $\begin{array}{l}\text { Cumple con la resolución } 2115 \text {, el valor máximo aceptado es de } \\
0.01 \mathrm{mg} / \mathrm{L} \mathrm{Pb} \text {, y el resultado está por debajo del límite de cuantificación } \\
\text { del instrumento con el que se realizó el ensayo. }\end{array}$ \\
\hline $\begin{array}{l}\text { Sólidos } \\
\text { suspendidos } \\
\text { Totales }\end{array}$ & $5 \mathrm{mg} / \mathrm{L}$ & \\
\hline Sólidos totales & $108 \mathrm{mg} / \mathrm{L}$ & \\
\hline
\end{tabular}

Fuente: Escuela de Ingenieros Militares (ESING)

\section{Resultados para ensayos realizados a la muestra de agua tomada el Campo Paradas Caldas.}

\section{Tabla 9}

Resultados calidad de agua de lluvia del Edificio Lara

\begin{tabular}{|c|c|c|}
\hline Ensayo & Resultado & Criterio \\
\hline Arsénico & $<0.005 \mathrm{mg} / \mathrm{L} \mathrm{As}$ & $\begin{array}{l}\text { Cumple con la resolución } 2115 \text {, el valor máximo aceptado es de } \\
0.01 \mathrm{mg} / \mathrm{L} \text { As, y el resultado está por debajo del límite de cuantificación } \\
\text { del instrumento con el que se realizó el ensayo. }\end{array}$ \\
\hline Cadmio & $<0.01 \mathrm{mg} / \mathrm{L} \mathrm{Cd}$ & $\begin{array}{l}\text { Cumple con la resolución } 2115 \text {, el valor máximo aceptado es de } \\
0.003 \mathrm{mg} / \mathrm{L} \text { Cd, y el resultado está por debajo del límite de cuantificación } \\
\text { del instrumento con el que se realizó el ensayo. }\end{array}$ \\
\hline Cobre & $<0.05 \mathrm{mg} / \mathrm{L} \mathrm{Cu}$ & $\begin{array}{l}\text { Cumple con la resolución } 2115 \text {, el valor máximo aceptado es de } \\
1.0 \mathrm{mg} / \mathrm{L} \mathrm{Cu} \text {, y el resultado está por debajo del límite de cuantificación } \\
\text { del instrumento con el que se realizó el ensayo. }\end{array}$ \\
\hline Coliformes Totales & $5.04 \times 10^{3} \mathrm{NMP} / 100 \mathrm{~mL}$ & $\begin{array}{l}\text { No cumple con el valor aceptable para agua potable de acuerdo al } \\
\text { decreto } 475 \text { de } 1998 \text {, y con la resolución } 2115 \text { de } 2007 \text {. }\end{array}$ \\
\hline Color & 68 UPC & $\begin{array}{l}\text { Esta característica física está por encima del valor máximo permisible en } \\
\text { la resolución } 2115, \text { por tanto no cumple el criterio de calidad de agua } \\
\text { potable, y debe tratarse para disminuir el color y está dentro de los } \\
\text { rangos permisibles. }\end{array}$ \\
\hline Cromo & $<0.05 \mathrm{mg} / \mathrm{L} \mathrm{Cr}$ & $\begin{array}{l}\text { Cumple con la resolución } 2115 \text {, el valor máximo aceptado es de } \\
0.05 \mathrm{mg} / \mathrm{L} \mathrm{Cr} \text {, y el resultado está por debajo del límite de } \\
\text { cuantificación del instrumento con el que se realizó el ensayo. }\end{array}$ \\
\hline D.B.O. 5 & $3 \mathrm{mg} / \mathrm{L} \mathrm{O} 2$ & \\
\hline D.Q.O. & $38 \mathrm{mg} / \mathrm{L} \mathrm{O} 2$ & \\
\hline
\end{tabular}




\begin{tabular}{|c|c|c|}
\hline Ensayo & Resultado & Criterio \\
\hline E. Coli & $\begin{array}{c}3.02 \times 10^{2} \\
\mathrm{NMP} / 100 \mathrm{~mL}\end{array}$ & $\begin{array}{l}\text { No cumple con el valor aceptable para agua potable de acuerdo al } \\
\text { decreto } 475 \text { de } 1998 \text {, y con la resolución } 2115 \text { de } 2007 \text {. }\end{array}$ \\
\hline $\mathrm{pH}$ & 6.72 Unidades de $\mathrm{pH}$ & \\
\hline $\begin{array}{c}\text { Sólidos } \\
\text { Sedimentables }\end{array}$ & $0.3 \mathrm{~mL} / \mathrm{L}$ & \\
\hline Temperatura & $18.0^{\circ} \mathrm{C}$ & \\
\hline Mercurio & $<0.002 \mathrm{mg} / \mathrm{L} \mathrm{Hg}$ & $\begin{array}{l}\text { Cumple con la resolución } 2115 \text {, el valor máximo aceptado es de } \\
0.001 \mathrm{mg} / \mathrm{L} \mathrm{Hg} \text {, y el resultado está por debajo del límite de } \\
\text { cuantificación del instrumento con el que se realizó el ensayo. }\end{array}$ \\
\hline Plomo & $<0.02 \mathrm{mg} / \mathrm{L} \mathrm{Pb}$ & $\begin{array}{l}\text { Cumple con la resolución } 2115 \text {, el valor máximo aceptado es de } \\
0.01 \mathrm{mg} / \mathrm{L} \mathrm{Pb} \text {, y el resultado está por debajo del límite de } \\
\text { cuantificación del instrumento con el que se realizó el ensayo. }\end{array}$ \\
\hline $\begin{array}{l}\text { Sólidos } \\
\text { suspendidos } \\
\text { totales }\end{array}$ & $29 \mathrm{mg} / \mathrm{L}$ & \\
\hline Sólidos totales & $128 \mathrm{~g} / \mathrm{L}$ & \\
\hline
\end{tabular}

Fuente: Escuela de Ingenieros Militares (ESING)

\section{Parámetros in situ}

Se realizó la medición de parámetros observados en el lugar obteniendo los siguientes resultados, ver tabla 10:

Tabla 10

Parámetros de calidad medidos en el laboratorio de la Escuela de Ingenieros

\begin{tabular}{|c|c|}
\hline Parámetro & Resultado \\
\hline Turbiedad & $16.47 \mathrm{FNU}$ \\
\hline $\mathrm{pH}$ & 6.99 \\
\hline TDS & $0.089 \mathrm{mg} / \mathrm{L}$ \\
\hline Resistividad & $4.8 \Omega^{*} \mathrm{~cm}$ \\
\hline Conductividad & $0.182 \mu \mathrm{s} / \mathrm{cm}$ \\
\hline Temperatura & $22.2^{\circ} \mathrm{C}$ \\
\hline
\end{tabular}

Fuente: Escuela de Ingenieros Militares (ESING)

\subsection{Tanque recoleccion de agua}

Actualmente el Cantón cuenta con con un tanque de $117 \mathrm{~m} 3$ ubicado en el edificio COING, capaz de capturar y almacenar el agua, sin embargo y teniendo en cuenta la aplicabilidad de la ecuación 15:

$$
\begin{gathered}
\frac{972.5 \mathrm{~m}^{3} / \mathrm{mes}}{25}=38.90 \mathrm{~m}^{3} / \text { día } \\
\frac{217 \mathrm{~m}^{3}}{38.90 \mathrm{~m}^{3} / \text { día }}=5,6 \text { dias }
\end{gathered}
$$

Se puede deducir que el agua obtenida podría abastecer los tres edificios para todas las labores por aproximadamente 5 días, teniendo en cuenta las 10 horas laborales diarias promedio en este estudio. Es por eso que se diseñaron dos prototipos de tanques que permitirían captar un volumen de $100 \mathrm{~m} 3$. Ver figura 4 y 5 . Se plantea para el caso de estudio un tanque auxiliar cilíndrico de $6,5 \mathrm{~m}$ de diámetro y $3 \mathrm{~m}$ de alto y su respectiva 
tapa sellable para permitir el ingreso en futuros mantenimeintos, cabe mencionar que de estar en funcionamiento los dos tanques se podrían llegar a captar y almacenar $217 \mathrm{~m}^{3}$ de agua aproximadamente.

Figura 4

Ubicación del tanque auxiliar y conexión con el tanque existente en el edificio COING

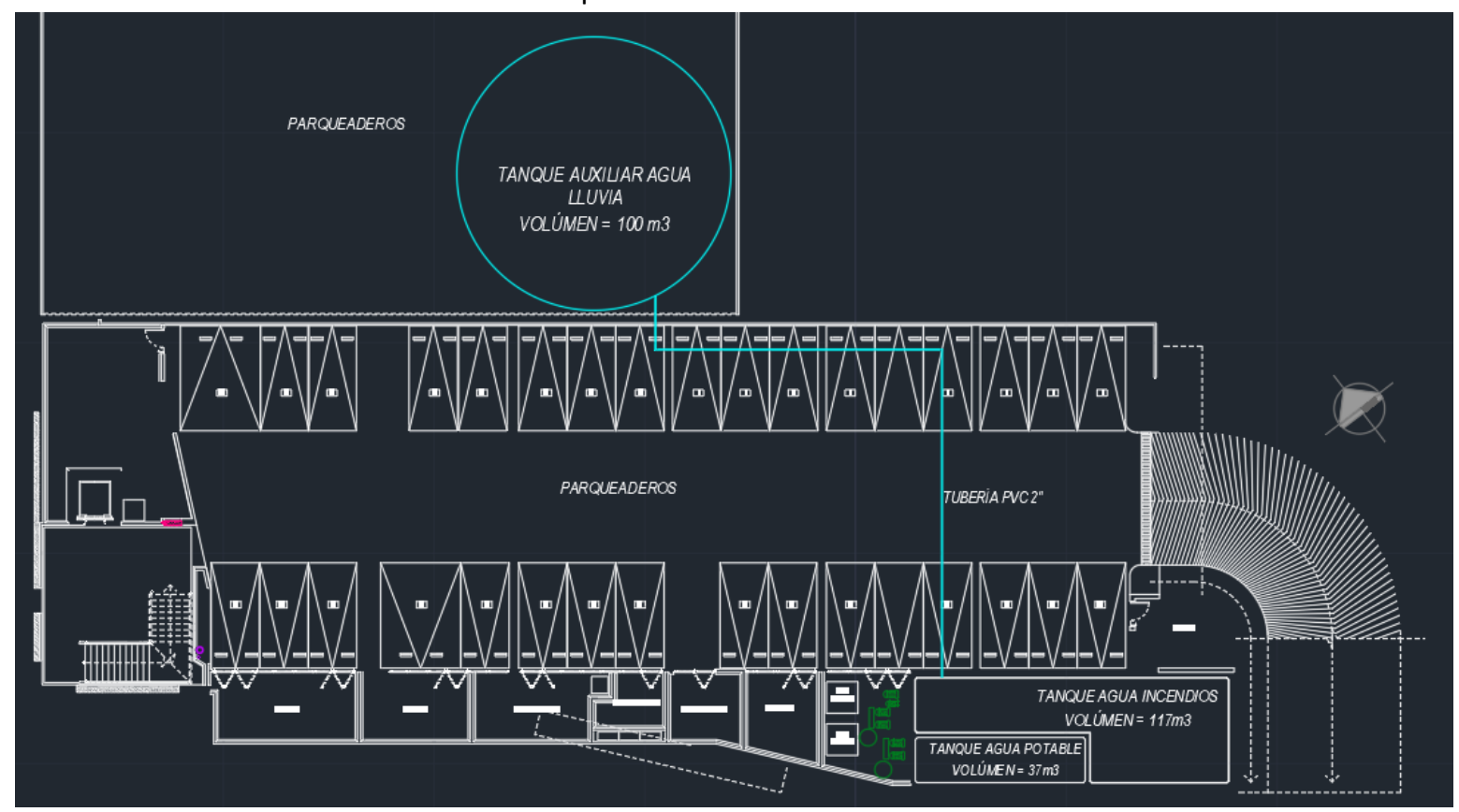

Fuente: Escuela de Ingenieros Militares (ESING)

Figura 5

Medidas del tanque cilíndrico auxiliar, $100 \mathrm{~m}^{3}$

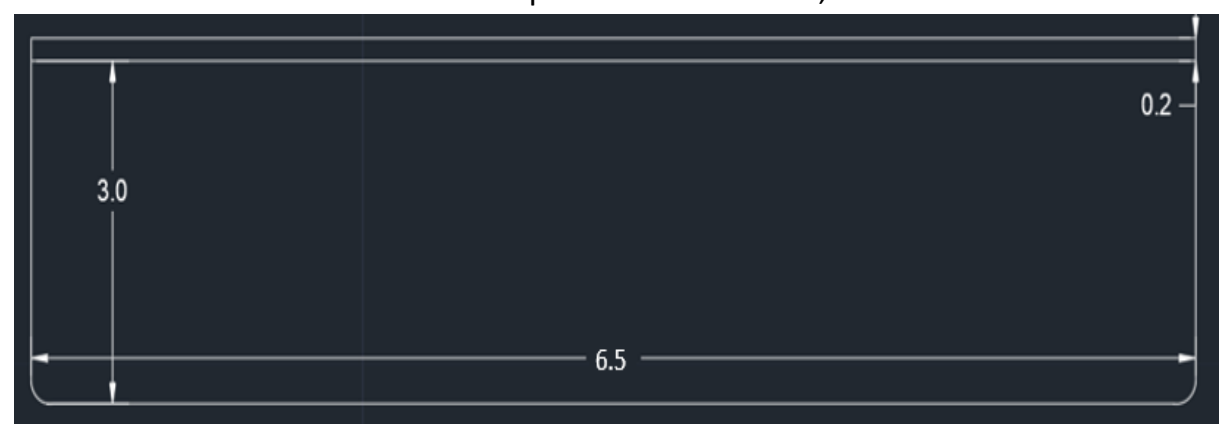

Fuente: Escuela de Ingenieros Militares (ESING)

Se debe tener en cuenta que el prototipo figura 5 cumple con los requisitos mínimos: todas las esquinas deben ir redondeadas para que el impacto del agua no genere que la estructura se fisure, se plantea que el material es concreto de $f^{\prime} c=28 \mathrm{MPa}$ reforzado con acero, incluso la tapa para que resista el peso que se le aplicará encima ya que es zona de parqueaderos, las paredes deben ir con refuerzo en acero o poner malla de gallinero para darle mayor seguridad.

\subsection{Posibles sistemas de tratamiento para agua de lluvia}

Si bien los análisis de fisicoquímicos fueron aceptables en el estudio, es necesario complementar mediante la utilzacion de sistemas de tratamiento para que finalmente el agua este apta para el consumo humano, si 
embargo el uso de esta es restringido pues debe estar bajo cierta normativa y fomentar el consumo de agua libre de agentes nocivos para la salud humana. Algunos de los tratamientos mas optimos se presentan a continuación:

Sistema con filtro de polietileno: Se hizo por una empresa en Betim, de Belo Horizonte Brasil; es un sistema que cuenta con cuatro (4) fases: filtra el agua para retirar partículas de tamaño grande, pasa por una piedra caliza ajustando el $\mathrm{pH}$, el agua pasa por cloro para eliminar microorganismos y potabilizar, por último, se pasa por un sistema de filtrado más fino. Cuando se cumpla con las 4 fases se puede llevar el agua al tanque de almacenamiento y disponer del agua almacenada.

Ecolibera: Es un depósito de agua de lluvia que permite que el agua captada en el techo, recorra a la bajante en donde tendrá un filtro con depósito exterior, que permite que el agua se limpie y se puede extraer por la llave que trae el depósito. Tiene una capacidad de aproximadamente 300 litros con una altura de $1.5 \mathrm{~m}$. Se tiene que tener en cuenta que al solo ser un filtro el agua no es apta para el consumo humano. Pero se consigue de una forma fácil en almacenes de cadena, de fácil instalación pero su uso es limitado por lo cual para este proyecto no es apto pero no deja de ser un buen equipo de depósito y tratamiento.

Por otro lado se encuentra el sistema Pure Water (Sistemas de Tratamiento Aguas de Lluvias): Creado por ARGEZA y consiste en sistemas de purificación de agua potable para tratar agua proveniente del acueducto, acueducto veredal, cuerpo de agua subterráneo o superficial y pluvial (Pure Water, 2019). Este sistema se ajustan según la capacidad requerida, calidad de agua de entrada y calidad de agua deseada. Utiliza un sistema de filtros llamados Rainy, que tiene características como un mecanismo de auto limpieza y se instalan en la pared conectándose a las bajantes de los techos, el agua entra al filtro el cual hace una rotación lenta hasta que pasa por todo el mecanismo con una velocidad específica, con la fuerza que se aplica se separan las partículas. El agua que sale y se almacena sirve para lavar vehículos, sanitarios, riego, lavadoras y la empresa cuenta con una línea de filtro más compuesto que permite potabilizar el agua para consumo humano. Los beneficios: eliminar impurezas que hay en los tejados y canaletas que dejan los animales, ahorro en el servicio de agua, sus equipos son de fácil traslado y mantenimiento. Ver figura 6.

Figura 6

Funcionamiento del filtro creado por ARGEZA.

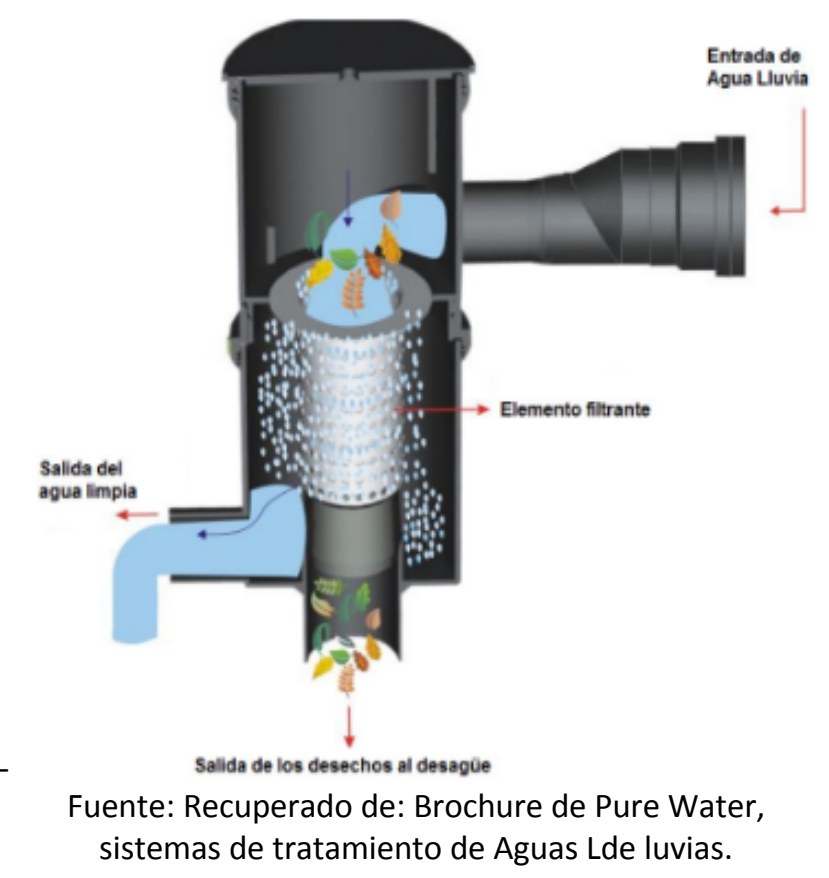


Esta opción fue la seleccionada como mejor sistema de tratamiento de agua de lluvia, puesto que asegura que potabiliza el agua, pero de igual forma se necesitaría un tanque de almacenamiento que debe cumplir con especificaciones y generaría un costo extra. Sin embargo es la opción más viable puesto que podría remplazar el consumo parcial de agua potable, ya que cada m3 suministrado por el acueducto tiene un valor de \$2999.65 pesos y el alcantarillado es considerado con un coeficiente de retorno de 0,85 del consumo de agua potable según Resolución 0330 de 2017 (Ministerio de Vivienda Ciudad y Territorio, 2017); el precio por m3 facturado por la empresa de acueducto es de $\$ 2544.56$ pesos. Como la facturación incluye a los tres edificios en un solo contador, el agua de lluvia que se puede ahorrar por mes equivale a $371.94 \mathrm{~m} 3 / \mathrm{mes}$, como lo evidencia la ecuación 16.

$$
\text { Ahorro mensual }=\left(371.94 \frac{\mathrm{m}^{3}}{m e s} * \$ 2999.5\right)+\left(\left(\frac{371.94 \mathrm{~m}^{3}}{m e s} * 0.85\right) * \$ 2544.56\right) \text { (16) }
$$

Al realizar un comparativo en precios del año 2018, se evidencia que el ahorro anual de agua potable reemplazada parcialmente por agua de lluvia, equivale a más de un mes de facturación, demostrado mediante la ecuación 17:

$$
\text { Ahorro anual }=\frac{\$ 1^{\prime} 920.094,13}{m e s} * 12 \text { meses }=\$ 23.041 .129,55 \text { año (17) }
$$

Es por eso que presentamos esta alternativa como la mejor opción, puesto que conjuga la utilidad frente al tema económico, pero es rentable y es una alternativa muy adecuada en términos generales.

\section{Conclusiones}

La selección del área de captación se basa en la cercanía que hay entre las edificaciones, la red de aguas de lluvia existente y los materiales de las cubiertas donde se plantea captar el agua de lluvia. No se seleccionaron otras áreas como parqueaderos, debido a que estas cuentan una red mixta de recolección de aguas delluvias, aguas grises y aguas negras, con lo cual la captación y tratamiento se hace mucho más difícil.

Para la correcta implementación de un sistema de aprovechamiento de aguas de lluvias se debe contar básicamente con 3 sistemas, los cuales son red de captación, planta de tratamiento, red de distribución, cabe resaltar que dentro del alcance del presente documento solo se hace énfasis en el primer sistema y se deja la localización del segundo sistema, con lo cual se recomienda que el desarrollo de los sistemas de tratamiento y red de distribución se haga en estudios independientes.

Esta propuesta conceptual del sistema de captación, no hace mayor énfasis en la cuantía de materiales, instalación y construcción de este, así mismo, se recomienda que para su construcción se realice un levantamiento topográfico el cual brinde mayor precisión en la ubicación de cada uno de los componentes de este sistema.

Se recomienda realizar una revisión y actualización de los planos hidrosanitarios de los edificios COING y ESING, ya que los planos que existen no concuerdan con la tubería que está instalada.

El proyecto abre campo en el área de la recolección y reutilización de aguas de lluvias en pro de generar procesos enmarcados al uso eficiente del recurso hídrico y por ende a disminuir problemáticas ambientales en que este se ve inmerso.

Los datos meteorológicos arrojan un acercamiento importante para entender las dinámicas climáticas de la zona de estudio, evidenciándose es un período bimodal, también ligado a los fenómenos de La Niña y El Niño, lo que nos demuestra que se pueden tomar medidas preventivas en pro de estos fenómenos de optimizar el consumo de agua de lluvia. 
Si bien el componente ambiental está enmarcado en el proyecto, es de tener en cuenta que existe un estímulo económico bastante grande pues el ahorro del recurso hídrico en el Cantón Caldas sería sustancial y beneficia al personal militar.

Para los análisis de aguas de lluvias obtenidas en el proyecto son necesarios monitoreos anuales pues precisan más los datos y de esta manera se podrían predecir más las capacidades a los tanques y por ende a la red hidrosanitaria.

Es necesario realizar otro análisis de aguas de lluvias pues estos fueron tomados en lugares inadecuados y arrojan niveles de coliformes que hacen imposible su uso humano, como resultado de una mala recolección de muestras en lugares no determinados.

Para poder implementar cualquiera de las opciones se debe construir un tanque de almacenamiento de agua auxiliar, que conecte a los tanques existentes de incendios pues son los que actualmente reciben agua de lluvia, dando como resultado un ahorro tanto en construcción como en procesos.

Se recomeinda que el tanque auxiliar sea subterráneo, ya que ninguno cuenta con bases para construir un tanque elevado, además se tendrían que hacer más procedimeintos para elevar el agua y esto incrementaría costos.

\section{Bibliografía}

Jaramillo, M. (2010). Potencial de reuso de agua residual doméstica como estrategia para el control de la contaminación por agua residual en el valle geográfico del río cauca. . Santiago de Cali: Universidad del Valle.

Ministerio de la proteccion social y de ambiente, v. y. (2007). Resolición numero 2115 de 2007. Bogotá: Ministerio de protección social .

Ministerio de Salud Publica. (1998). Decreto 475 de 1998. Bogotá: Ministerio de Salud Publica.

Ministerio de Vivienda Ciudad y Territorio. (2017). Resolución 0330 de 2017. Bogotá: Ministerio de Vivienda Ciudad y Territorio.

Palacio, N. (2010). Propuesta de un sistema de aprovechamiento de agua lluvia, como alternativa para el ahorro de agua potable, en la institución educativas María Auxiliadora de Caldas, Antioquia. En P. N, Propuesta de un sistema de aprovechamiento de agua lluvia, como alternativa para el ahorro de agua potable, en la institución educativas María Auxiliadora de Caldas. Medellin: Medellin.

Pure Water. (22 de 6 de 2019). Pure Water. Obtenido de https://purewater.com.co/sobre-nosotros/

Secretaria Distrital de Ambiente. (22 de 08 de 2007). Red de Monitoreo de Calidad del Aire de Bogotá. Obtenido de http://201.245.192.252:81/home/map 